\section{Unpräzision von Tag zu Tag}

R.-D. Hilgers ${ }^{1}$, N. Heussen ${ }^{1}$ und S. Stanzel ${ }^{2}$

${ }^{1}$ Institut für Medizinische Statistik, Universitätsklinikum der RWTH Aachen, Aachen, Deutschland

${ }^{2}$ Heidelberg, Deutschland

\section{Englischer Begriff imprecision from day to day}

Definition Die Unpräzision von Tag zu Tag beurteilt die Stärke der zufälligen Variabilität der Analyseergebnisse bei Bestimmung derselben Probe an mehreren Tagen mit derselben Messmethode.
Beschreibung Untersucht wird dieselbe Probe mit denselben Reagenzien und Geräten durch denselben oder verschiedene Analytiker unter möglichst wenig veränderten Umweltbedingungen.

\section{Literatur}

Qualitätskontrolle im Medizinischen Laboratorium von A bis Z - Ein Leitfaden in Schlagworten, 2. Aufl. Behring Diagnostika. https://doi. org/10.1515/labm.1995.19.1-12.218 